\title{
Evaluación de funciones ejecutivas en estudiantes de primer año universitario que presentan bajo y alto rendimiento en comunicación escrita y pro- ducción lectora
}

Evaluation of executive functions in first-year university students who have low and high performance in written communication and reading production

\author{
Paula Orozco Pineda \\ Unidad de Transversales \\ UNIMINUTO, Colombia
}

Edgar Pineda Martínez Corporación de Ciencia, Tecnología e Innovación para la Orinoquía

Colombia

\section{Resumen}

Este artículo presenta un estudio de evaluación de funciones ejecutivas en estudiantes de educación superior que presentaron bajo y alto rendimiento en prueba lectoescritora, con el fin de identificar causas que desde los procesos neurolingüísticas puedan afectar el rendimiento. Se realiza una investigación experimental con diseño de grupo control preprueba y posprueba aplicada a 202 estudiantes de primer año de educación superior. De los cuales, 101 tuvieron rendimiento bajo (grupo experimental) y 101 estudiantes rendimiento alto (grupo control). Se diseñó un programa de intervención en lectura y escritura para entrenar a los sujetos del grupo experimental. Se midió el uso de estrategias de aprendizaje, funciones ejecutivas y el desempeño lectoescritor de los sujetos antes de la intervención y de desempeño lectoescritor después de la intervención. Los resultados de las prepruebas evidenciaron bajos desempeños del grupo experimental frente al grupo control tanto en funciones ejecutivas como en estilos de aprendizaje. Se presentan los resultados de la posprueba aplicada al grupo experimental después de la intervención, mostrando mejoras significativas en el rendimiento. Se concluye con la necesidad de desarrollar programas de mejoramiento a las funciones ejecutivas enfocadas al proceso lectoescritor. 
Palabras clave: funciones ejecutivas, habilidades metalingüísticas, proceso lectoescritor, educación superior, estrategias de aprendizaje

\begin{abstract}
This article presents a study of evaluation of executive functions in higher education students who presented low and high performance in test writing and reading production, in order to identify causes that from neurolinguistics processes may affect the performance. Is an experimental research design of group control pretest and posttest applied to 202 freshmen of higher Education. Of these, 101 had under (experimental group) and 101 students yield high performance (control group). An intervention program was designed in reading and writing to train the experimental group subjects. Using strategies of learning, executive functions and performance writing and reading production of subjects was measured before the intervention and performance writing and reading production after the intervention. The results of the pre tests showed low performances of the experimental group compared to the group control in executive functions and learning styles. Presents the results of the posttest applied to the experimental group after the intervention, showing significant improvements in performance, allowing to conclude with the need to develop programmes of improvement to executive functions focused on the writing and reading production process.
\end{abstract}

Keywords: functions executive, metalinguistic skills, writing, reading production, higher education, strategies learning process

\section{Introducción}

$\mathrm{D}$ urante los últimos tres años, se vienen presentando aumento $\mathrm{e}$ índices de deserción universitaria. Las causas se han identificado de diversa índole; entre otras, se destacan factores económicos y sociales (Orozco y Pineda, 2017). Sin embargo, existe una razón poco identificada, esta radica en el rendimiento académico como detonante de la deserción estudiantil, lo que está asociado a bajos niveles de comprensión por parte de los estudiantes universitarios colombianos (Orozco y Pineda, 2017). Esta situación está especialmente situada en estudiantes que recién inician su aventura académica y el origen está en el desarrollo de problemas en lectura y escritura de índole académica.

Por tal razón, se creó la necesidad de desarrollar un proyecto de investigación que permitiera identificar las posibles causas asociadas a la lectura y escritura que puedan estar relacionadas con el bajo rendimiento académico, la deserción estudiantil y los bajos niveles de comprensión en estudiantes universitarios de primer año. Para tal efecto, se decide trabajar con población de primer año de once programas profesionales universitarios, cuatro de ellos de la modalidad presencial y siete programas de modalidad distancia de 
dos Instituciones de Educación Superior (IES) con presencia en la ciudad de Villavicencio, Colombia.

De esta manera, la selección de la población responde a cinco variables: 1. La ciudad de Villavicencio pertenece al departamento del Meta, una de las regiones con mayor reporte de deserción estudiantil en primer año universitario; 2. Los programas seleccionados responden a los de mayor reporte de deserción; 3. Son dos IES con mayor número de estudiantes de la región y ambas ofrecen programas en modalidad presencial y a distancia; 4. Ambas IES poseen programas de apoyo a los proceso de lectura y escritura para estudiantes; 5. Ambas IES poseen programas para mitigar la deserción estudiantil, teniendo el rendimiento académico como factor asociado a la problemática.

Por consiguiente, se decide desarrollar el proceso de diagnóstico, evaluación e intervención relacionados con las competencias escritas y procesos lectores como factor determinante e incidente en las problemáticas de deserción estudiantil universitaria. Además, se considera preponderante analizar desde la comprensión académica y el desarrollo de capacidades en lectura y escritura, determinantes para el éxito académico en la universidad, la transición entre educación media y educación universitaria. De esta manera, es claro que dicha transición genera cambios significativos en el comportamiento, conducta y actitudes hacia el estudio por parte de los estudiantes que ingresan a los programas universitarios.

Sin coordinador de disciplina, sin timbres, sin controles escolares, en la Universidad se descontrolan; no estudian lo suficiente, dejan de ir a clases, no organizan el tiempo y así, poco a poco, pueden ir teniendo problemas serios de rendimiento académico que cada vez los ponen en situación de riesgo de tener que abandonar la universidad (Camacho Díaz y Moreno Torres, 2004, p. 297).

Así mismo, y en correlación con la anterior problemática, se analizaron los resultados obtenidos por la IES en las pruebas Saber Pro $^{1}$ realizadas por el Instituto Colombiano para el Fomento de la Educación Superior (ICFES), en sus componentes de Evaluación del Lenguaje, cuyos elementos son la comprensión, la interpretación, el análisis y la producción de textos según necesidades y exigencias comunicativas de los contextos cultural-social y académico. De esta manera, en el nombrado análisis, se evidenciaron bajos niveles de puntuación y de logro, sobre todo en los niveles de lectura textual, inferencial y crítica, permitiendo de esta forma correlacionar las hipótesis de cambios significativos en comportamiento, conducta y actitudes hacia la deserción con bajos niveles de comprensión lectora y comprensión académica.

Esta hipótesis condujo a realizar un estudio que permitiera identificar el estado de los procesos de lectura y escritura a partir de su relación funcional con las habilidades metalingüísticas y los procesos cognitivos de los estudiantes, con el fin de identificar la incidencia de las funciones ejecutivas en el proceso de transición de la educación media con la educación universitaria. De esta manera, se podrán brindar oportunidad a dichos estudiantes de fortalecer sus competencias comunicativas para mejorar sus capacidades de comprensión académica y reforzar la 
formación integral que pueda subsanar los índices de deserción.

De esta forma, se inició en el año 2014 un proceso de diagnóstico e identificación de causas para bajos y altos rendimientos en lectura y escritura en estudiantes de primer año universitario. Para tal fin, se aplicó una prueba diagnóstica de competencias lectoras $\mathrm{y}$ procesos escriturales, denominada CEPLEC-V12 ${ }^{2}$, durante los últimos siete periodos académicos (2014-2, 2015-1, 2015-2, 2016-1, 2016-2, 2017 1, 2017-2). Así mismo, se han realizado encuestas a grupos de estudiantes evaluados, que por período académico han obtenido los puntajes más altos y más bajos en las pruebas. Además, a estos estudiantes se les ha aplicado la escala de valoración ACRA para identificación de estilos de aprendizaje y se han realizado una serie de pruebas neuropsicológicas sobre funcionalidad auditiva, visual, de lateralidad y de memoria, las cuales permiten dar una explicación más coherente del comportamiento desde las habilidades cognoscitivas y cómo estas están directamente relacionadas con las habilidades metalingüísticas y los proceso de lectura y escritura en el ámbito universitario (Orozco y Pineda, 2017).

En consecuencia, para efectos del presente estudio se identificaron 50 estudiantes, 25 con alto rendimiento y 25 estudiantes con bajo rendimiento presentado en las pruebas diagnóstico CEPLEC-V1, de los períodos 2015-1, 2015-2. A esta muestra, se le aplicaron los test y pruebas de funciones ejecutivas, el test ACRA de estilos de aprendizaje; este ejercicio se alternó con grupos focales y entrevistas estructuradas para establecer relaciones y correlaciones entre funciones ejecutivas, habilidades metalingüísticas, comunicación escrita y procesos lectores.

Así, el estudio se propuso responder la siguiente pregunta problema: ¿cuál es la relación entre las funciones ejecutivas y las habilidades metalingüísticas en una muestra de estudiantes de diferentes programas académicos que presentan bajo y alto rendimiento en pruebas de comunicación escrita y procesos lectores?

\section{Marco teórico}

Rendimiento en pruebas CEPLEC-V1. La prueba CEPLEC-V1 evalúa el nivel de competencia en comunicación escrita y procesos lectores de estudiantes universitarios entre 16 y 30 años, a través de ejercicios de lectura y escritura. La prueba ha sido desarrollada y aplicada durante los años 2014, 2015, 2016 y 2017 a estudiantes que ingresan a programas universitarios de pregrado. Está constituida por tres niveles de complejidad: el nivel uno hace referencia a ejercicios lectoescriturales textuales, el dos a ejercicios de inferencia y el tres se concentra en procesos de lectura y escritura de pensamiento crítico (Orozco y Pineda, 2016).

De esta manera, para medir y establecer rangos altos y bajos en el desarrollo de la prueba, se ha establecido que para el caso de la prueba CEPLEC-V1 el promedio mínimo es de 18 puntos, ponderables según el índice de puntaje global de las 30 preguntas de la prueba. Así, cada pregunta posee un puntaje mínimo y máximo según las claves establecidas y el nivel de lectura por evaluar: textual, inferencial y crítico. Por tal razón, los puntajes ubicados bajo 18 puntos se consideran con bajo rendimiento; 
por alto rendimiento, se entienden los puntajes que superan la media de 18 puntos (Pineda y Orozco, 2017).

\section{Comunicación escrita y procesos} lectores en estudiantes universitarios.

La comunicación escrita y los procesos lectores se configuran como herramientas primordiales en la formación académica universitaria, específicamente en el afianzamiento y consolidación de un pensamiento crítico y creativo (Sánchez, Mora, Duarte Acosta y Ortiz, 2014). De este modo, siguiendo a Lea (2004), la comunicación escrita y los procesos lectores, concebidos como una práctica social que puede variar según los contextos y las situaciones en las que se realiza, permiten revalidar posturas sobre el proceso de escritura: a) su aprendizaje es un proceso básico y b) la enseñanza de la escritura en la universidad es un proceso complementario concentrado en especialistas (Carlino, 2002). Por tal razón, se expone la emergencia de reconocer la relación existente entre formación disciplinar y competencias escritas y procesos lectores como un compromiso de la comunidad universitaria en el desarrollo de pensamiento crítico y creativo a través de la comprensión y producción de textos escritos (Carlino, 2004; Moreno, 2004).

Por consiguiente, se propone desarrollar un alfabetismo funcional de la lectura y la escritura en la universidad, desarrollando una relación sensible entre disciplinas, realidad circundante y comunicación escrita y procesos lectores a través de estrategias disruptivas que contribuyan en todas las asignaturas al desarrollo de pensamiento crítico y creativo. Ahora bien, un proceso de alfabetismo funcional va más allá de un ejercicio sistemático de lectura y escritura. Vincula en primera medida la necesidad de identificar el desarrollo de las funciones ejecutivas, reconocer si influyen o no en bajos rendimientos en competencias escritas y procesos lectores, definir protocolos de intervención y desarrollo de comprensión y producción de textos escritos desde un enfoque cognitivo, como lo propone Kabalen (2007).

Funciones ejecutivas. Estudiar las funciones ejecutivas y su influencia en procesos lectores es analizar el lenguaje y, por ende, hablar de cognición (Domínguez y Clemente, 1993). Es un proceso que genera más que comunicación y que logra integrar simbolismo, discurso y significado, es decir, lenguaje como la conglomeración de sistemas de la lingüística, la sintaxis, la semántica, la pragmática y la semiología. Además, es la posibilidad de entender el mundo, de apropiarse de este y de significarlo (Defior, 1996). Por tal razón, cuando hablamos de lenguaje, irremediablemente nos centramos en el cerebro como en el lugar específico donde las ideas, pensamientos, sueños e ilusiones se pueden materializar a través de la verbalización y la capacidad de darle sentido a la realidad.

Ahora, el estudio y evaluación de las relaciones existentes entre lenguaje y funciones ejecutivas es objeto relevante de diversas y variadas disciplinas configurándose como uno de los temas de mayor importancia en el desarrollo de disciplinas enfocadas en la lingüística, neurociencia, gramática, comunicación, filosofía y psicología, además de las múltiples variables y conexiones que entre estas disciplinas surjan. De esta manera, en lo correspondiente a los estudios y orientaciones de la 
presente investigación, es pertinente abordarlo desde la neuropsicología, en especial desde la neurolingüística, ya sea por su abordaje histórico o por la evaluación, abordaje y tratamiento de las funciones ejecutivas en estudiantes universitarios (Barceló Martínez, Lewis Harb y Moreno Torres, 2006).

De este modo, en la presente investigación se entienden las funciones ejecutivas como un conjunto de habilidades cognitivas que les permiten a los sujetos anticiparse a situaciones, diseñar y planear estrategias, planear operaciones mentales, autorregularse, monitorear tareas, seleccionar comportamientos, establecer conductas, flexibilizar los trabajos cognoscitivos, organizar el tiempo y el espacio: todo en función de la resolución de problemas (Pineda, 2000). Entonces, las funciones ejecutivas están relacionadas con acciones de los lóbulos frontales, en sus áreas prefrontales, así como de sus conexiones mutuas con otras zonas del córtex cerebral y otras estructuras subcorticales, lo que las hace directamente correlacionales con los procesos de aprendizaje.

Así mismo, Pineda (2000) afirma que las funciones ejecutivas les permiten a los sujetos la consecución de objetivos y la solución de problemas, así como de la capacidad de monitorear y autorregular el proceso para conseguir el objetivo o solucionar el problema. Por consiguiente, cuando existe una disfunción en dichos procesos se posibilita que el sujeto tenga problemas en su rendimiento en lectura y escritura, puesto que "el síndrome disejecutivo se debe a alteraciones del lóbulo frontal y se caracteriza por déficit de atención, fallas en la planificación y anticipación, déficit en las abstracciones y otras manifestaciones conductuales" (Castaño, 2003, p. 211).

Evaluación de las funciones ejecutivas. Para la presente investigación, se tuvieron en cuenta para ser susceptibles de evaluación las siguientes funciones ejecutivas: 1. planificación, 2. actitud selectiva y sostenida, 3. inhibición, 4. flexibilidad cognitiva, 5. fluidez verbal, 6. memoria de trabajo; además, 7. la funcionalidad visual, 8. la funcionalidad auditiva y 9. la lateralidad predominante en estudiantes universitarios. De esta manera, para evaluar la relación entre funciones ejecutivas y conducta académica en lectura y escritura de estudiantes universitarios, se exploró el funcionamiento neurocognitivo que propone Artigas (2009). De esta manera, se realiza la evolución de las funciones ejecutivas con el fin de cometer una exploración neuropsicológica que permitiera identificar fallas y alteraciones funcionales y de esta manera, poder diseñar intervenciones adecuadas para el grupo de universitarios evaluados.

Según Pineda (2000), las funciones ejecutivas pueden evaluarse desde la clínica cualitativa, la experimental y la cuantitativa. En esta investigación, se utilizó la evaluación cuantitativa o neuropsicológica. Esta es un tipo de evaluación que utiliza pruebas neuropsicológicas estandarizadas para una aproximación más objetiva. Este tipo de evaluación abarca, además de los aspectos cognitivos, aspectos sociales y afectivo-emocionales. Entonces, dentro de las pruebas más utilizadas para la evaluación de funciones ejecutivas se encuentran las pruebas de clasificación de tarjetas de Wisconsin (Wisconsin card shorting test), el test de fluidez verbal, la prueba de diseños, 
la organización del aprendizaje, la torre de Hanói o de Londres, el test de conflicto palabra color o la prueba de Stroop, el test ACRA y la prueba de apertura de caminos (Barceló Martínez, Lewis Harb y Moreno Torres, 2006): todas estas fueron aplicadas al grupo evaluado.

Habilidades metalingüísticas. Las habilidades metalingüísticas están relacionadas con la capacidad para describir, analizar, pensar y reflexionar sobre el sistema lingüístico, en su forma, contenido y uso del lenguaje en los diferentes contextos comunicativos, relacionados con procesos de alfabetización (Gombert, 1992; Kamhi, Lee y Nelson, 1985; Van Kleeck, 1995). Igualmente, para Chaney (1998) y Gombert (1992), el desarrollo de habilidades metalingüísticas depende íntimamente del desarrollo del lenguaje y, este a su vez, en el progreso de los procesos de lectura y escritura. Esta apreciación permite razonar que en los procesos de enseñanza de la lectura y la escritura es necesaria la toma de conciencia por parte de los individuos, del código lingüístico para poder analizarlo, pensarlo y reflexionarlo.

Por otra parte, para Van Kleeck (1994), las habilidades metalingüísticas conllevan dos tipos de conocimiento del lenguaje en procesos de lectura y escritura. El primero se refiere a la arbitrariedad y convencionalidad del lenguaje; la arbitrariedad referida al poder separar las palabras de las cosas que representan y la convencionalidad al establecer significados acordados por una comunidad. En este sentido, según Flórez et al. (2005), citando a Van Kleeck, la arbitrariedad del lenguaje comprende cuatro aspectos, ": (a) conciencia de palabra (diferenciación palabra-referente), (b) ambigüedad (múltiples significados), (c) sinonimia (palabras equivalentes) y, (d) lenguaje figurativo (significados no literales)" (p. 3). Así mismo, el segundo, refiere a entender el lenguaje como un sistema estructurado y sistemático constituido por sonidos, palabras en un ámbito fonológico, lingüístico y semántico constatable con su práctica de vida cotidiana donde se cargan de significado los sonidos y las palabras.

\section{Metodología}

La investigación tuvo un enfoque cuantitativo (Hernández, Fernández y Baptista, 2006). Su objetivo fue la explicación de los bajos niveles en lectura y escritura de estudiantes universitarios mediante el estudio de relaciones entre las variables de funciones ejecutivas y habilidades metalingüísticas. Se desarrolló un tipo de razonamiento hipotético-deductivo y se realizó un diseño experimental. La información fue recogida a través de pruebas estandarizadas y cuestionarios validados.

Así mismo, para el diseño de la investigación, se realizó un experimento con dos grupos de estudiantes, uno con rendimiento alto y otro con rendimiento bajo en competencia escrita y procesos lectores ${ }^{3}$. A estos, se les aplicaron pruebas estandarizadas y cuestionarios validados para funciones ejecutivas, con el fin de comprobar la existencia de relaciones causa-efecto entre rendimiento lectoescritor, funciones ejecutivas y habilidades metalingüísticas.

En ese sentido, se estableció que el diseño experimental fuera aleatorio, simple e intergrupal (grupo control y grupo experimental), para lo cual 
ambos grupos fueron objeto de la aplicación de la prueba CEPLEC-V1. Se organizaron los grupos homogéneos en número según rendimiento alto y bajo en las evaluaciones de la prueba, a ambos grupos se les aplicaron las pruebas estandarizadas y los cuestionarios validados. Sin embargo, solo el grupo experimental fue sujeto de un curso en lectura analítica y crítica para estudiantes universitarios, como proceso de intervención. Al final, se volvió a aplicar la prueba CEPLEC-V1 y se analizaron los resultados obtenidos.
Muestra. La prueba de CEPLECV1 fue aplicada a una totalidad de 1134 estudiantes de primer año universitario, de los programas en modalidad distancia tradicional y presencial de dos IES con presencia en la ciudad de Villavicencio. En consecuencia, el tamaño muestral con un $\mathrm{N}$ de 1134 estudiantes que pertenecen al tamaño del universo de estudiantes de primer año universitario de la totalidad de programas de pregrado que ofrecen las IES vinculadas. Para tal efecto, se aplica la siguiente fórmula:

Matriz de Tamaños Muestrales para diversos margenes de error y niveles de confianza, al estimar una proporción en poblaciones Finitas

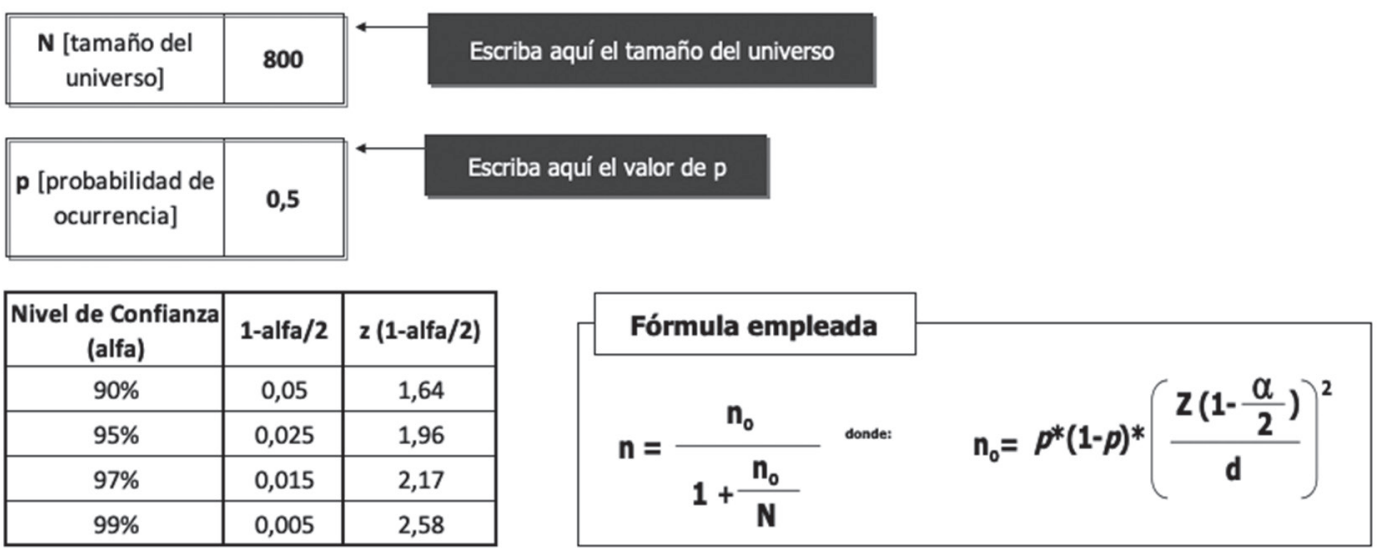

\begin{tabular}{|c|c|c|c|c|c|c|c|c|c|c|c|}
\hline \multicolumn{10}{|c|}{ Matriz de Tamaños muestrales para un universo de 800 con una p de 0,5 } \\
\hline \multirow{2}{*}{ Nivel de Confianza } & \multicolumn{10}{|c|}{ d [error máximo de estimación] } \\
\cline { 2 - 14 } & $\mathbf{1 0 , 0 \%}$ & $\mathbf{9 , 0 \%}$ & $\mathbf{8 , 0 \%}$ & $\mathbf{7 , 0 \%}$ & $\mathbf{6 , 0} \%$ & $\mathbf{5 , 0 \%}$ & $\mathbf{4 , 0} \%$ & $\mathbf{3 , 0 \%}$ & $\mathbf{2 , 0} \%$ & $\mathbf{1 , 0 \%}$ \\
\hline $\mathbf{9 0 \%}$ & 62 & 75 & 93 & 117 & 151 & 201 & 276 & 386 & 542 & 715 \\
\hline $\mathbf{9 5 \%}$ & 86 & 103 & 126 & 157 & 200 & 260 & 343 & 457 & 600 & 738 \\
\hline $\mathbf{9 7 \%}$ & 103 & 123 & 150 & 185 & 232 & 296 & 383 & 496 & 629 & 749 \\
\hline $\mathbf{9 9 \%}$ & 138 & 163 & 196 & 238 & 293 & 363 & 452 & 558 & 671 & 763 \\
\hline
\end{tabular}

Figura 1. Cálculo de la muestra poblacional.

Fuente: los autores. 
La muestra se estimó con un nivel de confianza de $95 \%$ y un error de estimación del 5\%. Por tal razón, estuvo conformada por un grupo de 202 estudiantes de primer año universitario. De los cuales, 101 de ellos presentaron rendimiento bajo y 101 rendimiento alto en las pruebas CEPLEC-V1. Para tal fin, se organizaron los puntajes de la prueba de mayor a menor y se seleccionaron los primeros y últimos 101 estudiantes. De los estudiantes con bajo rendimiento académico en competencias escritas y procesos lectores, el $67 \%$ pertenece a la modalidad virtual y a distancia, y el $33 \%$ pertenece a la modalidad presencial. La totalidad de estudiantes son de programas de pregrado (Gráfico 1).

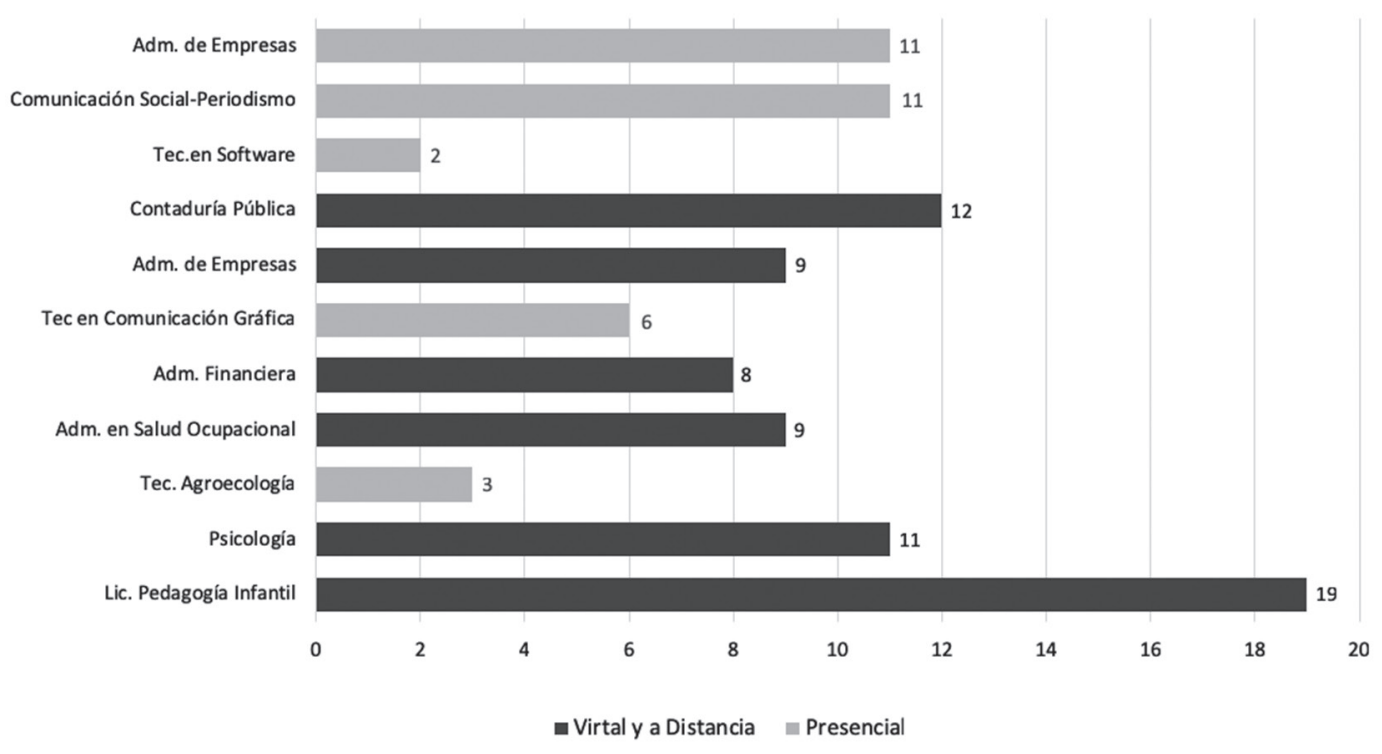

Gráfico 1. Número de estudiantes participantes con rendimiento bajo.

Fuente: los autores.

De los estudiantes con alto rendi- presencial. La totalidad de estudiantes miento académico en la prueba CE- pertenece a pregrado universitario PLEC-V1, 64\% es de modalidad vir- (Gráfico 2). tual y a distancia, y $36 \%$ de modalidad 


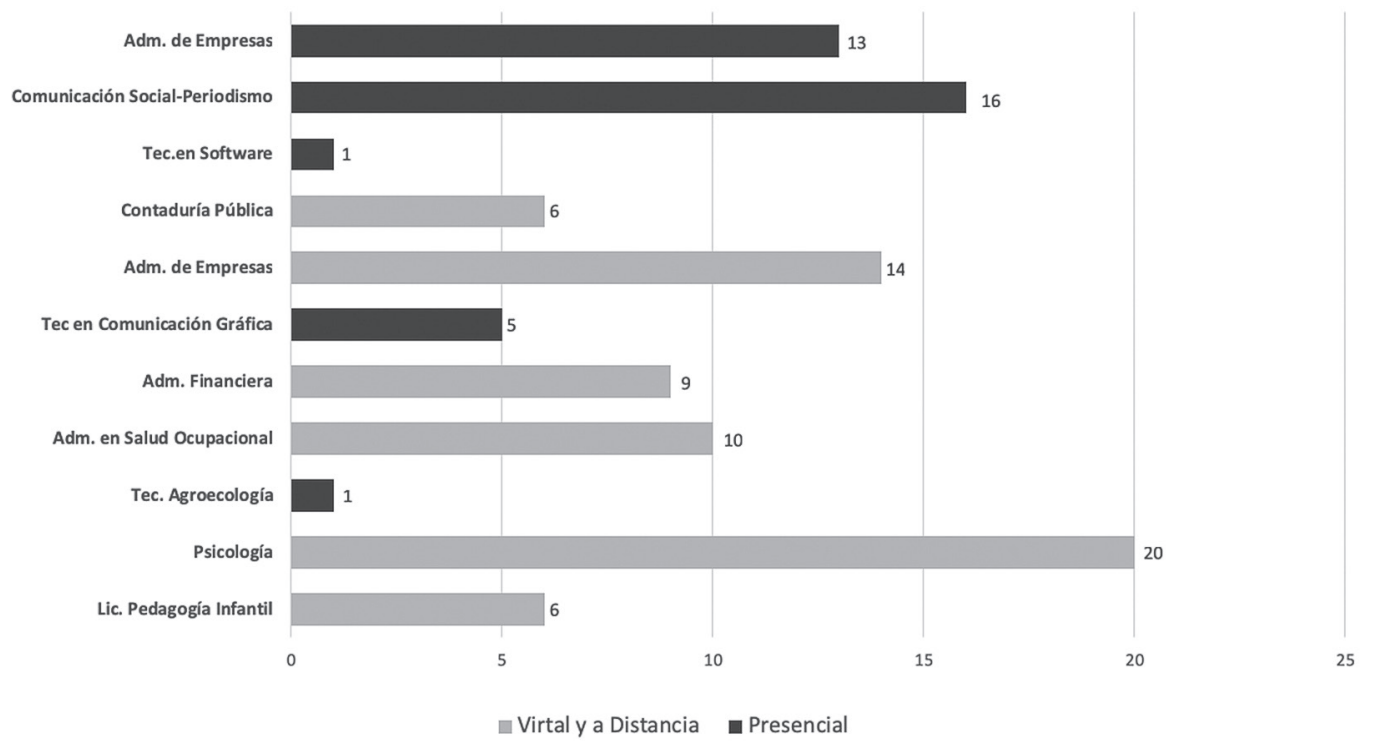

Gráfico 2. Número de estudiantes participantes con rendimiento alto.

Fuente: los autores.

La prueba CEPLEC-V1 fue aplicada un mismo día a todos los estudiantes (1131), en hoja y papel, en salones con condiciones adecuadas. Posteriormente, se dividieron en los dos grupos (control y experimental) y se aplicaron las pruebas de funcionamiento ejecutivo de manera individual y según cronograma establecido con cada uno de los participantes. Todas las pruebas se aplicaron en el mismo horario (9 a. m.), días entre semana y en instalaciones de las IES.

De esta forma, para evaluar las funciones ejecutivas en ambos grupos de estudiantes, se utilizó un protocolo que incluyó, en una primera sesión, una entrevista semiestructurada y la aplicación de los test: Wisconsin y ACRA. En una segunda sesión, se aplicó el de fluidez verbal y el Stroop. En tercera sesión, el Kaufman (Kbit). Al finalizar la aplicación de pruebas, se desarrolló un curso de lectura analítica y crítica para estudiantes universitarios de bajo nivel. En el curso, se realizaron ejercicios que potenciaron las funcionalidades visual, auditiva, lateralidad y de memoria (aplicado solo a grupo experimental). Posteriormente, se reunieron a los 202 estudiantes y se les aplicó nuevamente la prueba CEPLEC-V1.

\section{Instrumentos de evaluación}

Prueba CEPLEC-V1. La prueba CEPLEC-V1 es una prueba de fácil aplicación y corrección. Pretende evaluar el nivel de competencia en comunicación escrita y procesos lectores de estudiantes universitarios entre 16 y 30 años en diversas situaciones de lectura ${ }^{4}$ correspondientes al Marco Común Europeo de Referencia para las Lenguas (2001, 2002) y con diferentes tipos de textos ${ }^{5}$. 
La prueba ha sido baremada con una muestra de 3384 estudiantes pertenecientes a once programas universitarios diferentes, mostrando en los resultados niveles fiabilidad, homogeneidad y validez satisfactorias. Tiene una duración de 85 minutos y contiene 30 preguntas, que están cuidadosamente diseñadas para temáticas específicas y generales con el fin de evitar respuestas por conocimiento previo de los estudiantes.

La prueba CEPLEC-V1 está compuesta por siete textos, cuatro continuos y tres discontinuos, y por un total de 30 preguntas, elaboradas a la luz del marco teórico de PISA-2000. Los textos son continuos y argumentativos presentados en una sola columna y con numeración de las líneas de texto, se estiman en 250 y 475 palabras de longitud. En cuanto los textos discontinuos, se presentan en párrafos de no más de 200 palabras y van acompañados de gráficos, ejes cartesianos, gráficas y/o fotografías cada uno. Las 30 preguntas se clasifican en tres categorías según los aspectos básicos de la competencia lectora que evalúa PISA. La prueba incluye preguntas de puntuación, organización de textos, sintaxis y semántica, y recuperación de información. En cuanto al formato de respuesta, veintitrés ítems son de selección múltiple con cuatro alternativas, en los que solo una es correcta y tres son de formato abierto y demandan una respuesta breve del estudiante, dos son de organización secuencial, y dos son de puntuación de textos.

Entrevista semiestructurada. El objetivo de este instrumento era indagar de manera general aspectos familiares, sociales, académicos, médicos y personales que pudieran brindar información relevante al momento de analizar la escala de rendimientos y las pruebas de funcionalidad ejecutiva. Esta entrevista solo se realizó a los 202 estudiantes que hicieron parte de la muestra.

Test de clasificación de tarjetas de Wisconsin. Es el test de mayor uso para la evaluación de funciones ejecutivas. Se conoce con las siglas WCST. Busca evaluar la capacidad de abstracción, la formación de conceptos y las estrategias cognitivas de estudiantes universitarios para la solución de problemas y establecimiento de objetivos (Barroso-Martín y León-Carrión, 2002). Para el desarrollo del test, se requirió que los estudiantes respondieran frente a estímulos visuales a través de una clasificación categorial que deben deducir según la retroalimentación que se les presenta (Herrera, 2007).

Prueba de Stroop. Prueba que busca evaluar el control inhibitorio de la interferencia presentada; evalúa, además, la capacidad de inhibición a una respuesta automática. Por tanto, esta prueba contribuyó al presente estudio en el análisis de la respuesta de estudiantes universitarios frente a estímulos que se presentan en conflicto (Grodzinsky y Diamond, 1992). Esto con el fin de establecer parámetro de atención y respuesta. Para esta prueba, se requirió que los estudiantes leyeran los nombres de tres colores en un primer ejercicio; posteriormente, debían nombrar estímulos con cada uno de los colores; finalmente, en una tercera prueba, los estudiantes decían el nombre del color con el que estaba escrita la palabra, que, a su vez, es el nombre de otro color (Herrera, 2007).

Test de fluidez verbal. Este test se desarrolló a partir de dos tareas; 
en primer lugar, la tarea de fluidez verbal semántica (FVS), para lo cual se les solicitó a los estudiantes que dijeran el mayor número posible de palabras de la categoría "animales terrestres" durante un minuto y, en segundo lugar, la tarea de fluidez verbal fonológica (FVF), donde se les pidió a los estudiantes que enunciaran el mayor número posible de palabras que empezaran con las letras D, A y $\mathrm{M}$ durante un minuto; se realizan tres intentos cada uno con una letra diferente y se tabulan los tres resultados, la media de las tres da la variable FVF (Marino, Diaz-Fajreldines, 2011).

Para el desarrollo de ambas tareas, se advirtió y orientó a los estudiantes participantes que los nombres propios, las siglas, marcas comerciales y las palabras derivadas o verbos conjugados no se consideran como respuestas válidas. Este test evalúa la tarea de búsqueda de palabras y está asociado con el desarrollo y/o trastornos derivados de la conciencia fonológica en la lectura (Herrera, 2007).

Test breve de inteligencia de Kaufman (K-Bit). Es una prueba diseñada para la evaluación de la inteligencia, se concentra en la medición de la inteligencia verbal y no verbal en estudiantes universitarios. La prueba consistió en la aplicación de dos subtest, vocabulario y matrices. El subtest de vocabulario permite hacer una medida de la habilidad verbal, evaluando desarrollo del lenguaje, formación de conceptos verbales y caudal de información. El subtest de matrices mide habilidades no verbales y capacidad de solución de problemas a través del razonamiento por medio de estímulos visuales figurativos y abstractos (Barceló Martínez, Lewis Harb, Moreno Torres, 2006).
Para la prueba de vocabulario, en primer lugar, se les solicitó a los estudiantes nombrar objetos que se mostraban gráficamente; en seguida, se solicitaba el adivinar palabras a través de dos pistas suministradas. Para la prueba de matrices, los estudiantes debían elegir entre cinco figuras presentadas, la que mayor relación tuviera con la que se propone como estímulo abstracto.

Test ACRA. La escala de estrategias de aprendizaje ACRA tiene como finalidad identificar las habilidades de aprendizaje que son usadas con mayor frecuencia por los estudiantes en el momento de asimilación de información contenida en un texto. La prueba ACRA se compone de cuatro escalas independientes, que logran evaluar el uso de siete estrategias de adquisición de información, trece estrategias de codificación de información, cuatro estrategias de recuperación de información y nueve estrategias de apoyo al proceso (De la Fuente y Justicia, 2003).

Curso de lectura y escritura analítica y crítica. Curso diseñado desde las orientaciones del método de lectura analítico-crítica propuesto por Kabalen y De Sánchez (2003). El curso estuvo diseñado solamente para estudiantes quienes obtuvieron bajo rendimiento en la prueba CEPLEC-V1 y que fueron sujetos de evaluación de las pruebas de funciones ejecutivas. Por tal razón, se diseñó un curso para desarrollar la lectura comprensiva y una escritura de carácter analítico y crítica, para paralelamente ofrecer soluciones prácticas para superar las limitaciones y/o dificultades surgidas y evidenciadas en el desarrollo de las pruebas de funciones ejecutivas. 
Por tal razón, el curso integró los tres tipos de procesamiento de la información propuestos por Kabalen y De Sánchez (2003): nivel literal, nivel inferencial y nivel analógico, abordado desde diferentes grados de abstracción y complejidad. Se involucran de esta manera funciones ejecutivas como la visual, la auditiva, la atención, la memoria, el autocontrol, las emociones y la comprensión.

El curso tuvo una duración de cuatro semanas, en horarios intensivos de mañanaytarde.,Paracadainiciodesesión, se realizaban ejercicios neuromotores y de lateralidad, atendiendo a las necesidades de dicha función ejecutiva (Orozco y Pineda, 2017). Se realizaron los ejercicios propuestos por Kabalen y De Sánchez (2003), pero se ajustaron las lecturas de textos continuos y discontinuos a realidades contextuales y temporales de los estudiantes universitarios, se priorizó el trabajo de análisis y comprensión a través del uso de organizadores gráficos (esquemas, mapas, cartografías, diagramas, etc.); por último, se trabajaron ejercicios escriturales de tema libre y espontáneo.

\section{Resultados}

Se realizó una tabulación de los datos obtenidos de los test aplicados y se utilizó el paquete estadístico Statistical Package for Social Sciences (SPSS) para analizar frecuencias, relaciones y correlaciones de la información obtenida. A continuación, se presentarán tablas y gráficos referentes a cada prueba aplicada.

Prueba CEPLEC-V1. En las siguientes tablas se presentan los resultados de la prueba CEPLEC-V1 de los grupos de bajo y alto puntaje (Tabla 1 y Tabla 2, respectivamente). En la primera columna, se presenta el programa al que pertenecen los estudiantes; en la siguiente columna, el número de estudiantes que están en la categoría y la edad promedio; en la siguiente columna, se describen los estudiantes por género; en la siguiente columna se presenta el promedio de puntaje de la prueba en los tres niveles y total ${ }^{6}$; por último, se presenta la columna de modalidad del programa. 


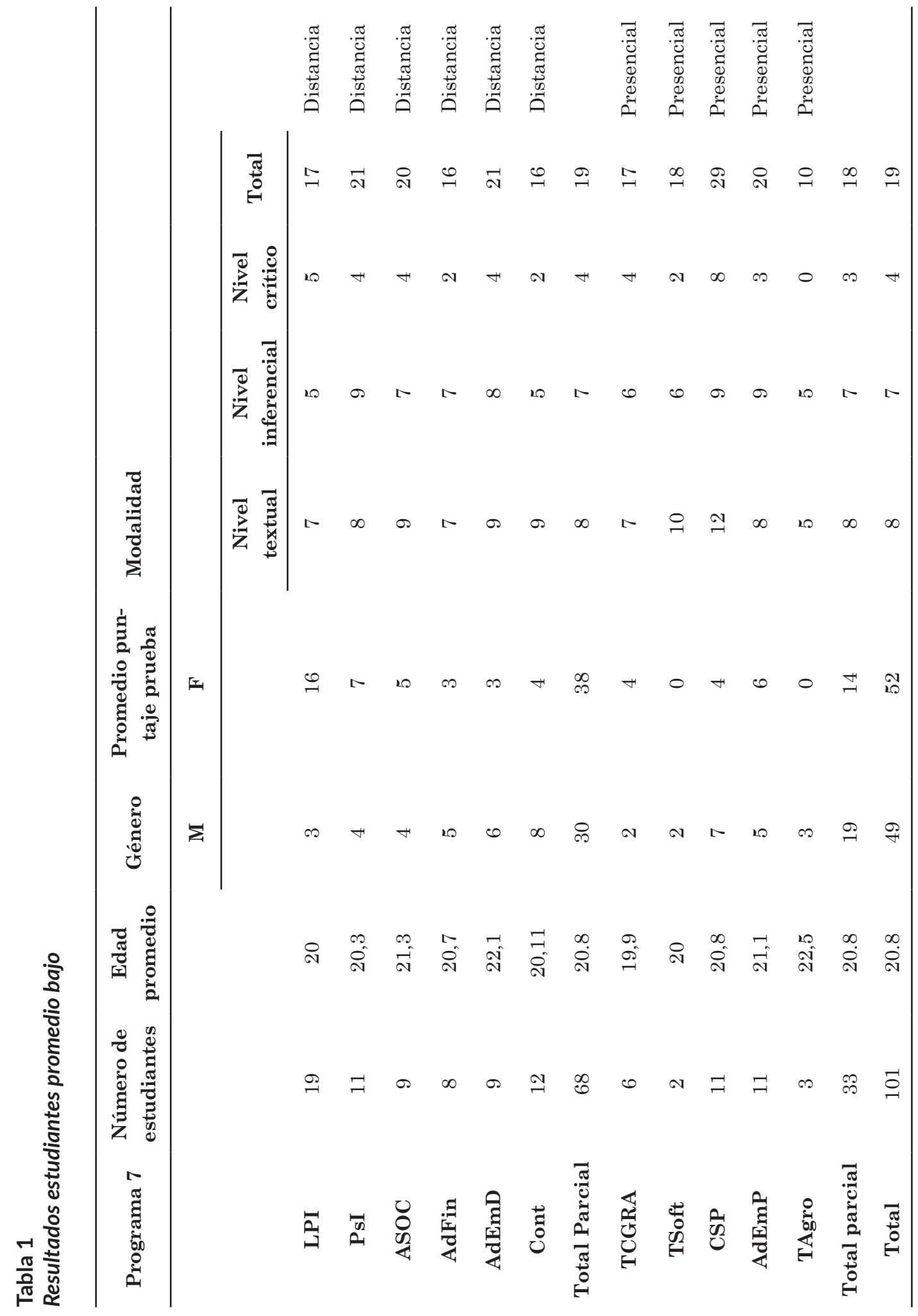




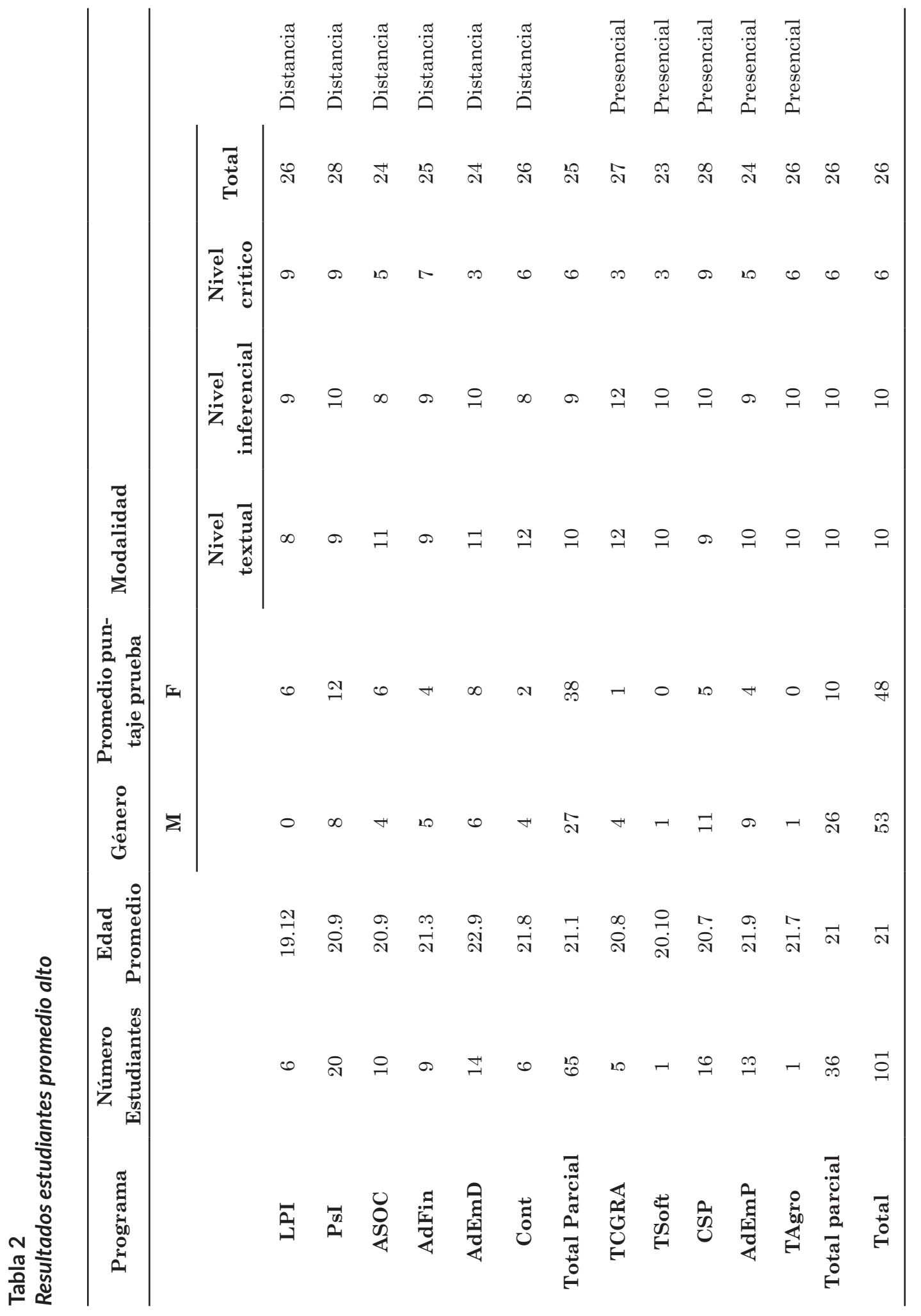


Entrevista inicial. A continuación (Tabla 3), se presentan los resultados obtenidos al realizar la codificación y clasificación de respuestas a la entrevista realizada a los 202 estudiantes de la muestra. Se discrimina en estudiantes con bajo nivel en la prueba CEPLEC-V1 (101) y estudiantes de nivel alto (101) en la misma prueba. En la primera columna se presentan las categorías resultantes de la codificación; en la segunda columna, el número de estudiantes correspondientes a cada categoría; y, en la tercera columna, los mismos datos de la segunda, pero en términos de porcentaje.

\section{Tabla 3}

\section{Resultados codificación entrevista inicial}

\begin{tabular}{|c|c|c|c|c|}
\hline Categoría & Número & Porcentaje & & \\
\hline & $\begin{array}{c}\text { Rendimiento } \\
\text { bajo }\end{array}$ & $\begin{array}{l}\text { Rendimiento } \\
\text { alto }\end{array}$ & $\underset{\text { bajo }}{\text { Rendimiento }}$ & $\begin{array}{c}\text { Rendimiento } \\
\text { alto }\end{array}$ \\
\hline \multicolumn{5}{|l|}{ Datos de identificación } \\
\hline Edad promedio & 20,8 & 21 & N.A. & N. A \\
\hline Género femenino & 52 & 48 & $51.5 \%$ & $47,5 \%$ \\
\hline Género masculino & 49 & 53 & $48.5 \%$ & $52.4 \%$ \\
\hline Hermanos promedio & 3 & 2 & N.A. & N. A \\
\hline $\begin{array}{l}\text { Lugar que ocupa entre los } \\
\text { hermanos, promedio }\end{array}$ & 2 & 2 & N.A. & N.A. \\
\hline Vive con ambos padres & 65 & 73 & $64.4 \%$ & $72.7 \%$ \\
\hline Vive con solo la madre & 24 & 19 & $23.7 \%$ & $18,8 \%$ \\
\hline Vive con solo el padre & 12 & 8 & $11.9 \%$ & $8 \%$ \\
\hline Madre fallecida & 6 & 8 & $6 \%$ & $8 \%$ \\
\hline Padre fallecido & 15 & 11 & $14.9 \%$ & $10.8 \%$ \\
\hline
\end{tabular}

Información sobre la madre

Edad promedio 


$\begin{array}{ccccc}\text { Empleada } & 28 & 33 & 27.8 \% & 32.6 \% \\ \text { Trabajo independiente } & 16 & 21 & 15.8 \% & 20.7 \% \\ \text { Ama de casa } & 34 & 27 & 33.6 \% & 26.7 \% \\ \text { Desempleada } & 9 & 5 & 8.9 \% & 5 \% \\ \text { Pensionada } & 8 & 7 & 7.9 \% & 7 \% \\ \text { Fallecida } & 6 & 8 & 6 \% & 8 \%\end{array}$

Máximo nivel académico

alcanzado

Primaria

56

24

$55.5 \%$

$23,7 \%$

Secundaria

34

50

$33.7 \%$

$50 \%$

Técnico

3

9

$2.9 \%$

$9 \%$

Profesional

2

8

$1.9 \%$

$8 \%$

Posgrado

0

2

$0 \%$

$2 \%$

Información sobre el padre

Edad promedio
Empleado
Trabajo independiente
Jefe de casa
Desempleado
Pensionado
Fallecido

59

57

N.A.

N.A.

54

38

$53.4 \%$

$37.6 \%$

15

40

$14.8 \%$

$39.6 \%$

2

1

$1.9 \%$

$1 \%$

9

4

$9 \%$

$4 \%$

6

7

$6 \%$

$7 \%$

Fallecido

15

11

$14.9 \%$

$10.8 \%$

Máximo nivel académico alcanzado 


\begin{tabular}{|c|c|c|c|c|}
\hline Primaria & 43 & 21 & $42.5 \%$ & $20.7 \%$ \\
\hline Secundaria & 35 & 52 & $34.6 \%$ & $52.3 \%$ \\
\hline Técnico & 6 & 6 & $6 \%$ & $6 \%$ \\
\hline Profesional & 2 & 8 & $2 \%$ & $8 \%$ \\
\hline Posgrado & 0 & 3 & $0 \%$ & $3 \%$ \\
\hline \multicolumn{5}{|l|}{$\begin{array}{c}\text { Información médica y } \\
\text { psicológica }\end{array}$} \\
\hline \multicolumn{5}{|l|}{$\begin{array}{l}\text { Enfermedades durante la } \\
\text { niñez }\end{array}$} \\
\hline Ninguna & 24 & 45 & $23.7 \%$ & $44.5 \%$ \\
\hline Media complejidad & 37 & 48 & $36.6 \%$ & $47.5 \%$ \\
\hline Alta complejidad & 30 & 8 & $29.6 \%$ & $8 \%$ \\
\hline \multicolumn{5}{|l|}{$\begin{array}{c}\text { Accidentes, cirugías o } \\
\text { lesiones }\end{array}$} \\
\hline Ninguna & 40 & 41 & $39.6 \%$ & $40.6 \%$ \\
\hline Media complejidad & 41 & 43 & $40.5 \%$ & $42.4 \%$ \\
\hline Alta complejidad & 20 & 17 & $19.8 \%$ & $17 \%$ \\
\hline Toma medicamentos & 28 & 15 & $27.7 \%$ & $15 \%$ \\
\hline No toma medicamentos & 73 & 86 & $72.3 \%$ & $85.8 \%$ \\
\hline $\begin{array}{l}\text { Asistencia a terapia } \\
\text { psicológica }\end{array}$ & 4 & 0 & $4 \%$ & $0 \%$ \\
\hline Exámenes neurológicos & 2 & 0 & $2 \%$ & $0 \%$ \\
\hline \multicolumn{5}{|l|}{$\begin{array}{c}\text { Antecedentes de } \\
\text { enfermedades familiares }\end{array}$} \\
\hline Ninguno & 11 & 9 & $10.8 \%$ & $9 \%$ \\
\hline
\end{tabular}


Baja complejidad

Media complejidad

Alta complejidad

Fumadores

Bebedores (Alcohol)

Rutinas de sueño
46

35

9

52

84

25

62

14

41

Anormales

Normales

Información académica

Pérdida académica en primaria

Pérdida académica en secundaria

Dificultades con la comprensión de lectura

Dificultades con las matemáticas

Rendimiento académico alto

Rendimiento académico medio

60

28

73

59

16

44
48

$45.5 \%$

$47.6 \%$

39

$34.6 \%$

$38.4 \%$

5

$9 \%$

$5 \%$

38

$51.4 \%$

$37.6 \%$

68

$83.1 \%$

$67.8 \%$

20

$24.7 \%$

$19.6 \%$

42

$61.3 \%$

$41.9 \%$

39

$13.8 \%$

$38.5 \%$

31

$40.5 \%$

$31 \%$

70

$59.4 \%$

$70 \%$

3

$14.8 \%$

$3 \%$

2

$27.7 \%$

$2 \%$

6

$72.2 \%$

$6 \%$

5

$58.4 \%$

$5 \%$

64

$15.8 \%$

$63.8 \%$

33

$43.5 \%$

$32.1 \%$ 


\begin{tabular}{|c|c|c|c|c|}
\hline $\begin{array}{l}\text { Rendimiento académico } \\
\text { bajo }\end{array}$ & 41 & 4 & $40.5 \%$ & $4 \%$ \\
\hline Estudió en colegio privado & 21 & 28 & $20.8 \%$ & $27.6 \%$ \\
\hline Estudió en colegio público & 80 & 73 & $79.2 \%$ & $72.4 \%$ \\
\hline \multicolumn{5}{|l|}{$\begin{array}{c}\text { Información } \\
\text { socio-económica }\end{array}$} \\
\hline Estrato 1 & 53 & 57 & $52.4 \%$ & $56.7 \%$ \\
\hline Estrato 2 & 38 & 42 & $37,5 \%$ & $41.3 \%$ \\
\hline Estrato 3 & 10 & 2 & $10 \%$ & $2 \%$ \\
\hline Estrato 4 & 0 & 0 & $0 \%$ & $0 \%$ \\
\hline Estrato 5 & 0 & 0 & $0 \%$ & $0 \%$ \\
\hline Estrato 6 & 0 & 0 & $0 \%$ & $0 \%$ \\
\hline Trabaja & 39 & 21 & $38.9 \%$ & $20.8 \%$ \\
\hline Cabeza de hogar & 13 & 18 & $12.7 \%$ & $17.8 \%$ \\
\hline Cabeza de familia & 16 & 14 & $15.8 \%$ & $13.9 \%$ \\
\hline
\end{tabular}

\section{Resultado funciones ejecutivas}

En la siguiente tabla, se presentan los promedios de resultados obtenidos por cada prueba evaluada. Para tal fin, en la primera columna, se ubica el nombre de la prueba; en la segunda, se ubica el rango promedio del grupo con bajo nivel en las pruebas CEPLECV1; en la tercera, el rango promedio del grupo de estudiantes con promedio alto en la prueba CEPLEC-V1; en la cuarta, se presenta el resultado de la prueba U de Mann- Whitney sobre los dos grupos; en la quinta, se presenta el nivel de significación exacta de carácter unilateral de ambos grupos, está representada por la letra P; y en la última columna, se presenta la comparación entre $\mathrm{P}$ y el nivel de significación (establecido en 0.05). 
Tabla 4

Resultados funciones ejecutivas

\begin{tabular}{|c|c|c|c|c|c|}
\hline Prueba $^{8}$ & $\begin{array}{c}\text { Rango promedio } \\
\text { grupo }\end{array}$ & $\begin{array}{c}\text { U de } \\
\text { Mann- Whitney }\end{array}$ & $\mathbf{P}$ & $\begin{array}{c}\text { P vs. S } \\
(0.05)\end{array}$ & \\
\hline & Bajo & Alto & $0-1$ & $0-1$ & $0-1$ \\
\hline WISCC & 2.84 & 5.21 & 2211.5 & .01 & $<0.05$ \\
\hline WISINT & 18.74 & 14.15 & 2093.5 & .04 & $<0.05$ \\
\hline WISRP & 13.82 & 8.26 & 1956 & .02 & $<0.05$ \\
\hline WISEP & 17.70 & 9.37 & 825 & .03 & $<0.05$ \\
\hline WISIC1C & 16.46 & 10.27 & 271 & .01 & $<0.05$ \\
\hline WISFMA & 22.07 & 9.60 & 120.5 & .04 & $<0.05$ \\
\hline STROOPP & 13.82 & 20.93 & 703.5 & .01 & $<0.05$ \\
\hline SRTOOPC & 14.25 & 21.25 & 811.5 & .01 & $<0.05$ \\
\hline STROOPPC & 13.94 & 20.94 & 763.5 & .02 & $<0.05$ \\
\hline K-BIT V & 12.67 & 23.01 & 58.5 & .04 & $<0.05$ \\
\hline K-BIT NV & 14.59 & 21.89 & 238 & .04 & $<0.05$ \\
\hline K-BIT CI_CO & 12.37 & 23.56 & 10210 & .02 & $<0.05$ \\
\hline FASFD & 14.46 & 22.48 & 10201 & .03 & $<0.05$ \\
\hline FASSA & 12.49 & 20.92 & 450.5 & .01 & $<0.05$ \\
\hline FASAM & 10.63 & 21.57 & 296.5 & .03 & $<0.05$ \\
\hline FASFPRPM & 12.53 & 21.66 & 5289.5 & .04 & $<0.05$ \\
\hline FASANIM & 12.01 & 20.65 & 117 & .01 & $<0.05$ \\
\hline FASFRUT & 14.32 & 21.43 & 815.8 & .01 & $<0.05$ \\
\hline FASEMPRPM & 13.16 & 21.04 & 466.4 & .04 & $<0.05$ \\
\hline
\end{tabular}


Finalmente, en la siguiente tabla tanto del grupo control (rendimiense presentan los resultados del Test to alto) como al grupo experimental ACRA aplicado a los estudiantes, (rendimiento bajo).

Tabla 5

Resultados Test ACRA

\begin{tabular}{|c|c|c|c|c|c|}
\hline Estrategias & $\begin{array}{c}\text { Rango } \\
\text { promedio } \\
\text { grupo }\end{array}$ & $\begin{array}{c}\text { U de } \\
\text { Mann- Whitney }\end{array}$ & $\mathbf{P}$ & $\begin{array}{c}\text { P vs. S } \\
(0.05)\end{array}$ & \\
\hline & Bajo & Alto & $0-1$ & $0-1$ & $0-1$ \\
\hline Adquisición de información & 30.47 & 45.96 & 61.5 & .48 & $>0.05$ \\
\hline Codificación de información & 34.43 & 44.60 & 60.54 & .17 & $>0.05$ \\
\hline Recuperación de información & 43.69 & 47.16 & 17 & .91 & $>0.05$ \\
\hline $\begin{array}{c}\text { Estrategia de apoyo de la } \\
\text { información }\end{array}$ & 35.49 & 48.22 & 33 & .27 & $>0.05$ \\
\hline
\end{tabular}

\section{Análisis}

En los resultados obtenidos en la entrevista (Tabla 3), se muestra que la edad promedio de los estudiantes oscila entre los 20 y 21 años, edad promedio alta para el común de inicio de carrera universitaria. Se estima que está 2 puntos encima de la media nacional y se analiza que están ingresando a la universidad tres años después de haber terminado estudios de bachillerato. Tienen en promedio dos hermanos y en general ocupan el segundo lugar entre ellos. Se presenta un mayor promedio de género femenino en el rendimiento bajo y de hombres en el rendimiento alto.

Así mismo, se nota una diferencia considerablemente alta entre los estudiantes que viven con ambos padres frente a los que viven solo con uno de ellos. En los estudiantes de bajo rendimiento, el $64 \%$ vive con sus padres y en los de rendimiento alto el $73 \%$ vive con ellos. Estos datos no son concluyentes y no permiten inferir la incidencia de hogares funcionales en el rendimiento bajo y/o alto de los estudiantes (Ver Tabla 3).

En cuanto a la información familiar, el promedio de edad de la madre está en los 50 años y el del padre en 58. Se supera en $70 \%$ el promedio de madres con trabajo y ocupación laboral (incluyendo amas de casa) y en $80 \%$ el promedio de padres con trabajo y ocupación laboral. El nivel de desempleo es bajo en ambos casos y no se presenta incidencia alguna significativa entre nivel ocupacional de los padres y rendimiento académico en lectura y escritura (Ver Tabla 3). 
En máximo nivel académico alcanzado, se denota una alta incidencia de padres que solo tienen primaria en estudiantes con bajo nivel en lectura y escritura. Hay mayor porcentaje de padres que alcanzaron nivel de secundaria en estudiantes con alto rendimiento en lectura y escritura. En el índice de educación terciaria, no se presentan datos significativos. Lo anterior puede evidenciar la importancia del nivel escolar del entorno familiar en el desarrollo de procesos en lectura y escritura acordes, ya sea en el apoyo y tutoría de los padres o en la capacidad de incidencia de estos en el potenciamiento de dichas habilidades (Ver Tabla 3)

Con relación a la información médica y psicológica, los resultados indican que el $23,7 \%$ y el $43.5 \%$ de los estudiantes con bajo y alto rendimiento en lectoescritura, respectivamente, manifestaron no haber presentado enfermedades en su niñez. El $40.5 \%$ y $42.4 \%$ de estudiantes con bajo y alto rendimiento, respectivamente, manifestaron haber presentado enfermedades de media complejidad relacionadas con el propio desarrollo de sus infancias. Sin embargo, el 29,6\% de los estudiantes del grupo de bajo rendimiento manifestó haber padecido enfermedades de alta complejidad durante su etapa infantil; dentro de estas enfermedades, las de mayor presencia fueron epilepsias, trastornos del desarrollo, parálisis de extremidades inferiores, 2 casos de artritis, un caso de distrofia muscular, un caso de pérdida auditiva, asma, polio y 6 casos de cáncer (dos de leucemia), 2 estudiantes son diagnosticados con TDAH (Ver Tabla 3).

En cuanto a accidentes, lesiones o cirugías, se presentan en ambos grupos lesiones o cirugías en la mayoría de casos por accidentes en casa y en juegos, como luxaciones y huesos partidos. Sin embargo, en los porcentajes de lesiones, accidentes o cirugías de alta complejidad en los dos grupos, se presentan demasiadas lesiones producidas por accidentes en trabajos de campo, caídas de caballos, caídas de árboles, golpes en la cabeza que predominan en un alto porcentaje en el grupo de bajo rendimiento en lectoescritura (Ver Tabla 3).

Así mismo, se analiza un porcentaje relativamente bajo de estudiantes que toman medicamentos en ambos grupos. Hay predominancia de medicamentos para el acné, anticonceptivos y en baja proporción medicamentos para problemas gástricos. Hay bajos resultados en asistencias a terapias psicológicas y/o a exámenes neuronales. En los datos familiares, se presentan enfermedades familiares centradas en obesidad, hipertensión, reumatismo y en baja proporción cáncer y otras enfermedades invasivas. No existen datos sobre enfermedades neuropsicológicas en el ámbito familiar de los estudiantes (Ver Tabla 3).

Es de notar, los altos índices de fumadores y bebedores de alcohol en el grupo de estudiantes de bajo rendimiento en lectoescritura. Estos son factores externos que deben ser evaluados en próximos estudios; así como los altos porcentajes en ritmos y rutinas de sueño y de alimentación que pueden generarse como factores asociados a los bajos rendimientos en lectura y escritura (Ver Tabla 3).

En general, según los datos obtenidos en la información médica y psicológica, no se muestran grandes diferencias entre los dos grupos. Las diferencias se encuentran en las lesiones, 
cirugías u operaciones que mostraron una mayor incidencia en estudiantes de bajo rendimiento en lectoescritura. Así mismo, la alta vulnerabilidad de procesos cognitivos y sociales a los que están sometidos los estudiantes del grupo de rendimiento bajo en lectoescritura en cuanto consumo de cigarrillo y alcohol, así como en rutinas de sueño y descanso.

Por otra parte, en los resultados obtenidos en el ítem de información académica, se muestra una alta diferencia entre los porcentajes de ambos grupos, mostrando un alto porcentaje de pérdidas académicas en los niveles de primaria y bachillerato en los estudiantes del grupo de rendimiento bajo. En cuanto la autopercepción sobre proceso en comprensión lectora, se denota un alto índice de poseer dificultades en lectoescritura por parte del grupo de estudiantes con bajo rendimiento. Estas percepciones se basan en pérdidas académicas, dificultades en lectura, bajo nivel de lectura y otros procesos asociados al ritmo y gusto lectoescritor, de la misma forma con el ítem de dificultades en matemáticas (Ver Tabla 3).

Estas autopercepciones se ven reflejadas en los procesos académicos, donde los estudiantes del grupo bajo presentaron bajos y medios niveles académicos; estos ítems se sustentaron en el análisis de informes de calificaciones de los grados octavo, noveno, décimo y undécimo facilitados por los estudiantes al proyecto. Este ítem refleja la incidencia entre rendimiento lectoescritor y proceso y logro académico. Es de anotar cómo en ambos grupos se presenta un predominio de estudiantes de colegios públicos frente a estudiantes de colegios privados (Ver Tabla 3).
En términos generales, en este ítem, sí se presentan diferencias significativas entre los grupos, siendo mejores los resultados en los estudiantes del grupo de alto rendimiento en lectoescritura. En el grupo de rendimiento bajo, se presentan altas pérdidas académicas, dificultades académicas evidenciadas en ausentismo escolar, pérdida académica, problemas comportamentales y gusto por el estudio, así como de las dificultades autopercibidas en lectura, escritura y matemáticas, resultados que evidencian la relación entre bajo rendimiento académico y bajos niveles de lectoescritura en estudiantes (Ver Tabla 3).

Así mismo, en los resultados obtenidos en la evaluación de funciones ejecutivas (Tabla 4), se presenta que en las pruebas donde se obtiene un valor de p. menor a 0.05 se debe rechazar la hipótesis de que ambos grupos son iguales y con un valor de $p$. mayor a 0.05 se acepta la hipótesis de que ambos grupos son iguales. Por tanto, y en términos de aplicación general de pruebas, se rechaza la hipótesis debido a que ninguna en el promedio general obtuvo un p. mayor al valor de significación de 0.05 (Ver Tabla 4).

Estos resultados permiten interpretar que sí existe una diferencia significativa en cuanto funciones ejecutivas y como estas, a través de los procesos cognitivos que coordinan, pueden incidir en el rendimiento lectoescritor de estudiantes de primer año universitario. Así mismo, las diferencias encontradas en los resultados del Test de ACRA (Tabla 5) permiten aceptar la hipótesis de que los grupos son diferentes en cuanto estrategias de estudio. Para esto, se analizan los datos en adquisición, recuperación, codificación 
y apoyo a la información, demostrando que en el grupo de rendimiento lectoescritor bajo hay fuertes falencias en tener una estrategia de estudio claro. Esto evidencia la duración promedio en la aplicación de la prueba CEPLECV1, la cual está diseñada para ser contestada en un período de tiempo comprendido entre 45 y 50 minutos; para el grupo de rendimiento, bajo en lectoescritura el promedio de tiempo para la prueba fue de 57,23 minutos.

Por otra parte, las explicaciones al bajo rendimiento en lectoescritura por parte de estudiantes de primer año universitario podrían estar relacionadas con posibles déficits en las funcionalidades ejecutivas que repercuten en procesos cognoscitivos, conductuales y emocionales. Así, los resultados comparados muestran diferencias significativas en los puntajes obtenidos por el grupo de rendimiento bajo frente a los estudiantes del grupo de rendimiento alto en lectoescritura, según la prueba CEPLEC-V1. Por otra parte, el test de clasificación de tarjetas de Wisconsin presentó niveles bajos y medios en la capacidad de abstracción, la formación de conceptos y las estrategias cognitivas de los estudiantes universitarios del grupo de rendimiento bajo, estos procesos se consideran básicos en el proceso lectoescritor e inciden notablemente en el desarrollo de habilidades metalingüísticas y procesos cognitivos del ejercicio lectoescritor (Flórez, 2013).

Para la prueba del Test de Stroop, se presentan puntajes bajos en los componentes evaluados en torno a la capacidad de inhibición a una respuesta automática por parte de los estudiantes del grupo de rendimiento bajo en lectoescritura. Presentan conflictos entre la atención sostenida y la posible respuesta, elementos esenciales en procesos de comprensión lectora (Grodzinsky y Diamond, 1992). Por ende, el bajo rendimiento lectoescritor podría tener relación con el funcionamiento ejecutivo, sobre todo en los niveles de conceptualización, abstracción del pensamiento, memoria sostenida, concentración y respuesta oportuna.

Así mismo, las diferencias entre ambos grupos presentadas en las pruebas de fluidez verbal y semántica se puntualizan en los puntajes básicos del grupo de bajo rendimiento en problemas de conciencia fonológica y posesión de conceptos, velocidad de pensamiento y bajo procesamiento de información, todas estas categorías necesarias para el desarrollo de comprensión escrita y procesos lectores (Orozco y Pineda, 2017). Las pruebas, también mostraron diferencias significativas en las pruebas de matices; se presentan bajos niveles en habilidades verbales, con bajo desarrollo de aptitudes en inferencias y analogías, conceptos verbales y conocimiento de palabras. Se muestra una posible incidencia de problemas del lenguaje en los estudiantes con bajo nivel lectoescritor.

De esta forma, los resultados también evidenciaron una posible relación entre bajo rendimiento lectoescritor, disfuncionalidades ejecutivas y antecedentes familiares, psicológicos, académicos y socioeconómicos de los estudiantes. En este sentido, tardía inserción a la vida académica, lesiones, cirugías y golpes en la niñez, consumo de licor y cigarrillo, bajos niveles de logro académico en primaria y bachillerato, bajo nivel académico de los padres, ausencia de terapias psicológicas y/o psicopedagógicas, inexistencia de 
programas de apoyo académico en el proceso lectoescritor, estratos socioeconómicos bajos y vulnerables pueden probar que en entornos vulnerables la incidencia de bajos niveles de funciones cognitivas está más latente y presente, convirtiéndose en una dificultad significativa para enfrentar las diferentes exigencias académicas y sociales en el ámbito universitario.

\section{Discusión}

Los resultados obtenidos mostraron la importancia de realizar pruebas de funcionalidades ejecutivas a estudiantes de primer año universitario, tanto para entender y hacer seguimiento a los perfiles cognoscitivos de los estudiantes, como para entender dificultades académicas que en muchos casos sobrepasan los estigmas de la voluntad y el ánimo, y se centran en verdaderos problemas cognoscitivos y/o conductuales con base neuronal.

Así mismo, los resultados obtenidos por ambos grupos de estudiantes permiten establecer programas de mejoramiento e intervención que permitan en mínimo tiempo ir solventando y superando las disfuncionalidades ejecutivas en estudiantes de primer año universitario. Por tal razón, la presente investigación presentó la oportunidad de desarrollar un curso de intervención desde las orientaciones del método de lectura analítico-crítica propuesto por Kabalen y De Sánchez (2003); para el desarrollo de la lectura comprensiva y la escritura de carácter analítico y crítica, que paralelamente ofrezca soluciones prácticas para superar las limitaciones y/o dificultades surgidas y evidenciadas en el desarrollo de las pruebas de funciones ejecutivas.

En este sentido, el curso tuvo una duración de cuatro semanas, en horarios intensivos de mañana y tarde. Para cada inicio de sesión se realizaban ejercicios neuromotores y de lateralidad, atendiendo a las necesidades de dicha función ejecutiva (Orozco y Pineda, 2016). Se realizaron los ejercicios propuestos por Kabalen y De Sánchez (2003), pero se ajustaron las lecturas de textos continuos y discontinuos a realidades contextuales y temporales de los estudiantes universitarios, se priorizó el trabajo de análisis y comprensión a través del uso de organizadores gráficos (esquemas, mapas, cartografías, diagramas, etc.), y, por último, se trabajaron ejercicios escriturales de tema libre y espontáneo. Al término del curso, se aplicó nuevamente la prueba CEPLEC-V1 a los estudiantes que tomaron el curso, presentando los siguientes datos: 


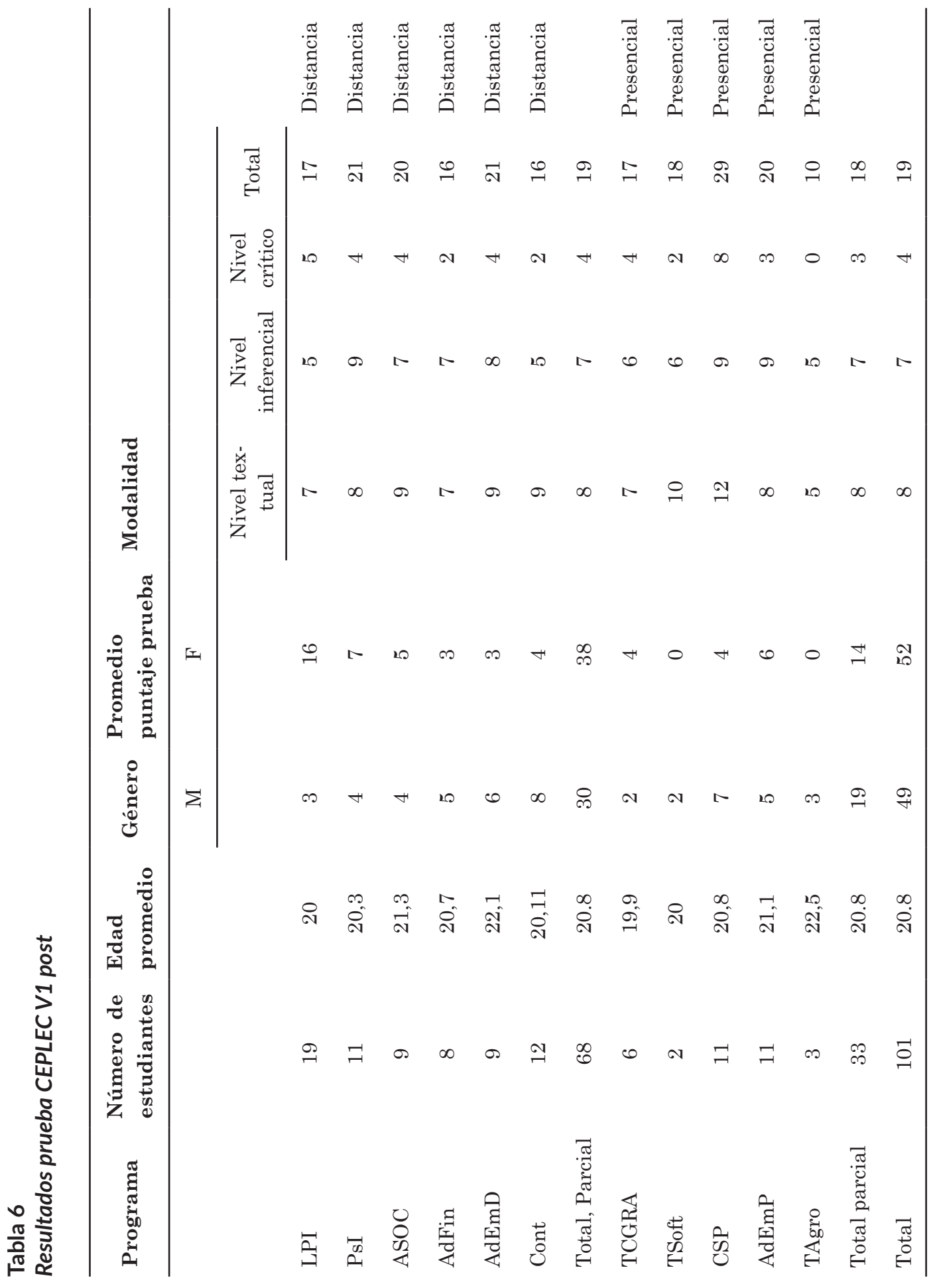


Al analizar los resultados, se evidencia la mejora en la mayoría de programas, alcanzando y sobrepasando el umbral de 18 puntos en seis de ellos y mejorando el promedio de puntaje inicial en todos los demás. Se concluye la eficacia de un programa de mejora en competencias lectoescriturales desde la potencialización de funciones ejecutivas. Estos resultados permiten a su vez pensar en la posibilidad de evaluar en funciones ejecutivas nuevamente al grupo de estudiantes con el fin de establecer posibles mejoras en los procesos cognitivos. Esta evaluación se ha establecido para un año después del presente ejercicio, con el fin de evaluar la sostenibilidad de la estrategia y de las pautas de lectoescritura ofrecidas en el proceso de intervención.

En este sentido, las competencias escritas y procesos lectores se convierten en el propósito central de la formación universitaria. Se convierten en una necesidad de alfabetización emergente que propende por el alto rendimiento académico y se convierte en un instrumento esencial para el aprendizaje y el desarrollo de otros saberes y competencias en el ámbito académico. Así mismo, la formación y/o alfabetización emergente en competencias escritas y procesos lectores no deben asumirse como actividades complementarias de las disciplinas universitarias en pregrado, sino que deben ser elementos constitutivos de ellas. En este punto, cabe la pregunta sobre el nivel y/o desarrollo de procesos lectoescritores en docentes disciplinarios en el ámbito universitario ¿Cuál es el nivel y/o calidad lectoescritora de docentes universitarios?

De esta forma, las IES no solamente se debe preocupar por el fomento de programas para fortalecer la lectura y la escritura, sino que deben ahondar en las causas del nivel de los estudiantes en el proceso lectoescritor. Según los datos del estudio, las causas además de variadas pueden llegar a ser complejas. La incidencia del desarrollo de funciones ejecutivas, el nivel socioeconómico, el ámbito familiar, la historia médica inicial y familiar, y los estilos de aprendizajes son elementos fundamentales y claves para el diseño de programas de alfabetización emergente en lectura y escritura en la universidad.

Por tal razón, es preponderante que las IES posean programas eficaces de evaluación y abordaje de competencias escritas y procesos lectores. Así mismo, debe propiciar cursos y estrategias de mejoramiento y perfeccionamiento de habilidades y capacidades comunicativas a lo largo del proceso académico universitario. Esto permitirá llevar un control significativo del desarrollo e incidencias de las funciones ejecutivas en los procesos de comprensión y rendimiento académico, y mejorar las capacidades de pensamiento y lectura crítica. Lo anterior permite reducir los altos porcentajes de deserción estudiantil en cuanto se mejoran los procesos de acompañamiento académico y se brindan posibilidades de evaluación holística y formativa desde un enfoque de desarrollo humano inclusivo y equitativo.

\section{Notas}

1. Examen y prueba estandarizada de Estado aplicada al final de la carrera universitaria.

2. Batería de pruebas de comprensión lectora para estudiantes universitarios, pruebas creadas por los autores y que actualmente está en proceso de validación por pares externos. 
3. Según prueba CEPLEC-V1.

4. Personal, pública, profesional, educativa.

5. Continuos y no continuos.

6. Todo puntaje total menor a 18 es considerado bajo, todo puntaje mayor a 24 es considerado alto.

7. $\quad \mathrm{LPI}=$ Licenciatura en Pedagogía Infantil; Psi=Psicología, $\mathrm{ASOC}=$ Administración en salud ocupacional; AdFin=Administración Financiera; AdEM=Administración de Empresas Distancia; Cont= Contaduría pública; TCGRA=Tecnología Comunicación Gráfica; TSoft=Tecnología en desarrollo de software; CSP=Comunicación Social y Periodismo; AdEmp=Administración de Empresas presencial; TAgro: Tecnología en agroecología.

8. WISCC: Test Wisconsin -categorías completadas; WISINT: Test Wisconsin - intentos para completar la prueba; WISRP: Test Wisconsin - respuestas perseverativas; WISEP: Test Wisconsin - errores perseverativas; WISIIC: Test Wisconsin - intentos para completar la primera categoría; WISFMA: Test Wisconsisn - fallos para mantener la atención; STROOPP: Test Stroop Palabras; STROOPC: Test Stroop Palabras-Color; STROOPPC: Test Stroop Palabras-color o interferencia; K-BIT V: Prueba de Vocabulario; K- BIT NV: Prueba de Matrices; K-BIT Cl_CO: compuesto, Diferencia entre las puntuaciones obtenidas en la prueba de Vocabulario y de Matrices; FASFD: Prueba de FAS Letra D total; FASSA: Prueba de FAS Letra A total; FASAM: Prueba de FAS Letra M total; FASFPRPM: Promedio Prueba FAS fonológico; FASANIM: Prueba FAS animales; FASFRUT: Prueba FAS frutas; FASEMPRPM: Promedio Prueba FAS semántico.

\section{Bibliografía}

Artigas-Pallarés, J. (2009). Modelos cognitivos en el trastorno por déficit de atención/hiperactividad. En Revista de Neurología, 49(11).

Barceló Martínez, E., Lewis Harba, S. y Moreno Torres, M. (2006). Funciones ejecutivas en estudiantes universitarios que presentan bajo $\mathrm{y}$ alto rendimiento académico. En Psicología desde el Caribe, 18.

Barroso-Martín, P.M. y León-Carrión, J. (2002) Funciones ejecutivas: Control, planificación y organización del conocimiento. En Revista de Psicología General y Aplicada, 55(1).

Camacho Díaz, A. y Moreno Torres, M. (2004) Retos del aseguramiento de la calidad. En El futuro de la formación en Ingeniería. XXIV Reunión Nacional de Facultades de Ingeniería. Cartagena de Indias.

Carlino, P. (2002). Enseñar a escribir en todas las materias: cómo hacerlo en la universidad. Ponencia invitada en el Panel Enseñanza de la Escritura, Seminario Internacional de Inauguración de la Subsede Cátedra UNESCO, Instituto de Lectura y Escritura, Faca. de Educación, Univ. Nac. de Cuyo, Mendoza, abril de 2002. En http:// www.educ.ar/educar/superior/biblioteca_digital/ (2004). El proceso de escritura académica: cuatro dificultades de la enseñanza universitaria. Educere, 26(8). En: http://www.unne.edu.ar/ institucional/documentos/lecturayescri - tura08/paula_carlino.pdf

Castaño, J. (2003) Trastornos del aprendizaje. Los caminos del error diagnóstico. Unidad de Neuropediatría, Departamento de Pediatría, 
Hospital Italiano, Buenos Aires, Argentina. En Arch.arget.pediatr.

Chaney, C. (1998). Preschool language and metalinguistic skills are linked to reading success. En Applied psycholinguistics, 19.

De la Fuente, J. y Justicia, F. (2003). Escala de estrategias de aprendizaje. ACRA-Abreviada para alumnos universitarios. Revista Electrónica de Investigación Psicoeducativa y Psicopedagógica, 1.

Defior, S. (1996). Las dificultades de aprendizaje: un enfoque cognoscitivo. Málaga: Aljibe.

Díaz-Fajreldines, H, Marino, J. (2011). Pruebas de Fluidez Verbal Categoriales, Fonológicas y Gramaticales en la Infancia: Factores Ejecutivos y Semánticos. En Revista Chilena de Neuropsicología, 6. En http://www.redalyc.org/articulo. oa?id=179322533008

Domínguez, A.B. y Clemente, M. (1993). ¿Cómo desarrollar secuencialmente el conocimiento fonológico? En Comunicación, Lenguaje \& Educación, 19(21).

Flórez R. (2013). Programa de mejoramiento de las competencias comunicativas de estudiantes de la Facultad de Medicina: un estudio piloto. Bogotá: Universidad Nacional.

Flórez, R., Torrado, M.C., Arévalo, I., Mesa, C., Mondragón, S. y Pérez, C. (2005). Habilidades metalingüísticas, operaciones metacognitivas y su relación con los niveles de competencia en lectura y escritura: un estudio exploratorio. Forma y Función (18).

Gombert, J. (1992). Metalinguistic development. Chicago: University of Chicago.

Grodzinsky, G. M. y Diamond, R. (1992). Frontal lobe functioning in boys with attention-deficit hyperactivity disorder. Developmental Neuropsychology, 8.

Hernández, R., Fernández, C. y Baptista, P. (2006). Metodología de la investigación. Bogotá: Mac Graw Hill.

Herrera Pino, J. (2007). Manual de examen neuropsicológico de la Demencia. United States: Academy of Applied Cognitive Neuroscience.

Kabalen D.A. (2007). Análisis y Pensamiento crítico para la expresión verbal. Monterrey: Editorial digital ITESM.

Kabalen, D.A. y De Sánchez, M. (2003). La lectura analítico crítica. Un enfoque cognoscitivo aplicado al análisis de la información. México: Trillas.

Kamhi, A., Lee, R. y Nelson, L. K. (1985). Word syllable and sound awareness in language disordered children. En Journal of speech and hearing disorders, 50.

Lea, M.R. (2004). Academic literacies: a pedagogy for course design. En Studies in Higher Education, 29(6).

Moreno, M. (2004). El lenguaje en la educación superior. En FlórezRomero, R. (ed.). El lenguaje en la educación. Una perspectiva fonoaudiológica, 283-327. Bogotá: Facultad de Medicina, Universidad Nacional de Colombia, Unibiblos.

Orozco, P., Pineda, E. (2017). Comunicación escrita, procesos lectores y habilidades metalingüísticas de estudiantes de primer año universitario. Estudio de Caso. En Revista Internacional de Aprendizaje en la Educación Superior, 3(2).

Pineda, D. (2000) La función ejecutiva y sus trastornos. En Revista de Neurología, 30(8). En: http://www.revneurol.com/3008/ i0800764.pdf 
Sánchez, N., Mora, N., Duarte, J. y Or- Van Kleeck, A. (1995). Emphasizing form tiz, C. (2014). Tejedores de Comunicación: Comprender y producir textos académicos en UNIMINUTO. Bogotá: Corporación Universitaria and meaning separately in rereading and early reading instruction. En Minuto de Dios. 
